\title{
Cryptococcus laurentii Aplicado em Pós-Colheita Reduz Podridões em Maçãs
}

\author{
Luiz E. B. Blum ${ }^{1}$, Cassandro V. T. Amarante², Rosa M. Valdebenito-Sanhueza ${ }^{3}$, Letícia S. Guimarães², \\ Alexandre Dezanet ${ }^{2} \&$ Pedro Hack Neto $^{2}$
}

'Departamento de Fitopatologia, Universidade de Brasília, CEP 70910-970, Brasília, DF, e-mail: luizblum @ unb.br; ${ }^{2}$ Departamento de Fitotecnia, Centro de Ciências Agroveterinárias, Universidade do Estado de Santa Catarina, Cx. Postal 281, CEP 88502-970, Lages, SC; ${ }^{3}$ EMBRAPA Uva e Vinho, Cx. Postal 130, CEP 95700-000, Bento Gonçalves, RS

(Aceito para publicação em 17/02/2004)

Autor para correspondência: Luiz E. B. Blum

BLUM, L.E.B., AMARANTE, C.V.T., VALDEBENITO-SANHUEZA, R.M. GUIMARÃES, L.S., DEZANET, A. \& HACK NETO, P. Cryptococcus laurentii aplicado em pós-colheita reduz podridões em maçãs. Fitopatologia Brasileira 29:433-436. 2004.

\section{RESUMO}

As podridões pós-colheita podem ocasionar perdas substanciais em maçãs (Malus domestica). O mofo azul (Penicillium expansum), a podridão amarga (Glomerella cingulata) e a podridão olho-de-boi (Pezicula malicorticis) estão entre as mais comuns. Grande atenção tem sido dada ao uso de alternativas de controle às doenças pós-colheita. A aplicação pós-colheita de leveduras, como o Cryptococcus laurentii, é uma das opções. Neste estudo testou-se a eficiência de $C$. laurentii (isolado 36) para o controle de podridões em maçãs 'Fuji' e 'Gala'. Após aplicação dos produtos, através de imersão, os frutos foram armazenados em laboratório $\left(15-20{ }^{\circ} \mathrm{C} / 60-70 \%\right.$ UR) ou em câmara fria $\left(1{ }^{\circ} \mathrm{C} /\right.$ $90-95 \%$ UR). Os patógenos foram inoculados na concentração de $10^{2}$ conídios $\mathrm{ml}^{-1}$, a levedura a $10^{7}$ células $\mathrm{ml}^{-1} \mathrm{e}$ os fungicidas a
$150 \mathrm{mg} \mathrm{l}^{-1}$. Em testes de laboratório, a aplicação de $C$. laurentii reduziu as podridões (G. cingulata, $P$. expansum e $P$. malicorticis) da maçã tanto quanto os fungicidas testados (thiabendazol e iprodione). Nos testes efetuados em câmara fria, constatou-se que, após o armazenamento, o tratamento de maçãs com $C$. laurentii foi tão eficiente quanto os tratamentos com fungicidas (thiabendazol, iprodione, digluconato de clorohexidina, dicloro-s-triazinatriona sódica, dicloroisocianurato de sódio e hipoclorito de sódio) na redução da podridão causada por $P$. expansum. Aplicação de $C$. laurentii não alterou a firmeza de polpa nem o teor de sólidos solúveis totais ( $\left.{ }^{\circ} \mathrm{Brix}\right)$ dos frutos.

Palavras-chave adicionais: Glomerella cingulata, Malus domestica, Penicillium expansum, Pezicula malicorticis.

\section{ABSTRACT}

Post harvest application of Cryptococcus laurentii reduces apple fruit rots

Rots might be responsible for substantial post harvest losses in apples (Malus domestica). The blue mold caused by Penicillium expansum, the bitter rot caused by Glomerella cingulata, and the 'bull's-eye rot' induced by Pezicula malicorticis are among of the most common rots. Great attention has been given to less environmental damaging alternatives for the control of postharvest diseases. The post harvest application of east-like fungi, such as Cryptococcus laurentii, is one option for controlling fruit rot. This study was carried out to test the efficiency of $C$. laurentii on fruit rot control in 'Fuji' and 'Gala' apples. After application of treatments, by immersion, the fruits were stored in the laboratory $\left(15-20{ }^{\circ} \mathrm{C} / 60\right.$ $70 \% \mathrm{RH})$ or in cold storage $\left(1{ }^{\circ} \mathrm{C} / 90-95 \% \mathrm{RH}\right)$. The pathogens were applied at a concentration of $10^{2}$ conidia $\mathrm{ml}^{-1}$, the yeast at $10^{7}$ cells $\mathrm{ml}^{-1}$, and the fungicides at $150 \mathrm{mg} \mathrm{l}^{-1}$. The Cryptococcus laurentii was as efficient in reducing apple fruit rots ( $G$. cingulata, P. expansum, and $P$. malicorticis) as were the fungicides thiabendazole and iprodione. In cold storage trials, $C$. laurentii was as efficient as the fungicides (thiabendazole, iprodione, chlorhexidine digluconate, sodium dichloro-s-triazinetrione, sodium dicloroisocyanurate, and sodium hypochloride) in reducing $P$. expansum fruit rot. The application of $C$. laurentii did not affect flesh firmness and the total soluble solids content ( ${ }^{\circ}$ Brix) of the fruits.
A cultura da macieira (Malus domestica Borkh.) é de grande importância econômica para o estado de Santa Catarina. O estado é o maior produtor brasileiro da cultura, com uma produção na safra 2001/2002 de cerca de 400 mil toneladas (ABPM, 2002). Todavia, perdas substanciais da produção de maçãs resultam de podridões após a colheita dos frutos. As podridões causadas por Glomerella cingulata (Stonem) Spauld. \& Schrenk (podridão amarga), Penicillium expansum Link. (mofo azul) e Pezicula malicorticis (H. Jacks.) Nannf. (podridão olho-de-boi) em pós-colheita estão entre as mais importantes (Boneti et al., 1999; Blum et al., 2000).
O controle pós-colheita de doenças em maçãs geralmente é feito com a imersão de frutos em soluções fungicidas. Entretanto, a redução no uso de fungicidas é uma preocupação mundial. O controle biológico de doenças em pomáceas tem mostrado resultados satisfatórios (Janisiewicz \& Roitman, 1998; 1990; Valdebenito-Sanhueza \& Cattanio, 2001). Este trabalho foi conduzido com o objetivo de avaliar a eficiência da levedura Cryptococcus laurentii (Kufferath) Skinner no controle pós-colheita de podridão amarga, mofo azul e podridão olho-de-boi em maçãs, cvs. Gala e Fuji.

Para obtenção de inoculo, P. expansum, G. cingulata e 
P. malicorticis foram cultivados em BDA, sob temperatura ambiente $\left(15\right.$ a $\left.20^{\circ} \mathrm{C}\right)$, durante sete, dez e 14 dias, respectivamente. Cryptococcus laurentii (isolado 36), selecionado por Valdebenito-Sanhueza \& Cattanio (2001), foi cultivado por $72 \mathrm{~h}$ em BDA.

Os testes em laboratório, cujos produtos estudados estão apresentados na Tabela 1 , foram delineados em blocos acaso, com oito repetições de 20 frutos cada. Inicialmente, os frutos da cv. Fuji foram desinfetados em hipoclorito de sódio ( $1 \%$ de cloro ativo) durante 3 min e então perfurados com alfinetes próximos à região equatorial (quatro furos eqüidistantes de $1 \mathrm{~mm}$ de diâmetro e $1 \mathrm{~mm}$ de profundidade). Em seguida, os frutos foram imersos durante 15 min nas suspensões compostas de água esterilizada infestada com os patógenos (concentração de inoculo ajustada para $10^{2}$ conídios dos patógenos $\mathrm{ml}^{-1}$ ). Em tais suspensões foram, então, aplicados os fungicidas ou a levedura (concentração ajustada para $10^{7}$ células $\mathrm{ml}^{-1}$ ). Em cada experimento foi incluído um tratamento testemunha onde somente a levedura foi aplicada, no entanto, este tratamento e seus resultados não foram apresentados (Tabela 1) por não ter sido usado na análise estatística. Posteriormente à aplicação dos produtos, os frutos foram acondicionados em bandejas de papelão e incubados em sala de ambiente escuro a $15-20{ }^{\circ} \mathrm{C} / 60-70 \%$ UR. Após o aparecimento dos sintomas iniciais, diariamente foram avaliados a incidência $(\%)$ e o diâmetro das lesões (mm) ocasionadas pelo patógenos. Para simplificar a apresentação dos dados (Tabela 1), foram apresentados apenas os resultados referentes às avaliações efetuadas aos dez dias para $P$. expansum, 22 dias para $G$. cingulata e aos 30 dias para $P$. malicorticis.

Os quatro testes (três com a cv. Fuji e um com a cv. Gala), em câmara fria comercial, (Tabela 2) foram delineados em blocos acaso, com seis repetições de 20 frutos cada. Inicialmente, os frutos (cvs. Fuji e Gala) foram submetidos aos mesmos procedimentos descritos para os testes de laboratório. No entanto, as suspensões foram preparadas em água esterilizada infestada com $P$. expansum (concentração de inoculo ajustada para $10^{2}$ conídios $\mathrm{ml}^{-1}$ ), nas quais, foram misturados os fungicidas ou a levedura (concentração ajustada para $10^{7}$ células $\mathrm{ml}^{-1}$ ). Em cada experimento foi incluído um tratamento testemunha onde somente a levedura foi aplicada, no entanto, este tratamento e seus resultados não foram apresentados (Tabela 1) por não ter sido usado na análise estatística. Posteriormente à aplicação dos produtos, os frutos foram acondicionados em bandejas, que foram colocadas em caixas comerciais de papelão (capacidade para $18 \mathrm{~kg}$ de frutos), e, então, armazenados a $1{ }^{\circ} \mathrm{C} / 90-95 \%$ UR em câmara fria (Empresa Yakult S.A., Lages/SC). Após um período de 40 a 90 dias de armazenamento em câmara fria, dependendo do experimento (Tabela 2), os frutos foram avaliados imediatamente e sete dias após a retirada da câmara fria, quanto à incidência de doença $(\%)$, o diâmetro das lesões $(\mathrm{mm})$ ocasionadas pelo patógeno, a firmeza de polpa $\left(\mathrm{Kg} \mathrm{F} \mathrm{cm}^{-2}\right) \mathrm{e}$ os teores de sólidos solúveis totais ( $\left.{ }^{\circ} \mathrm{Brix}\right)$.

Comprovada a significância dos dados através da Análise de Variância, as médias dos tratamentos foram comparadas entre si através do teste de Tukey $(\mathrm{P}=5 \%)$.

Nos testes efetuados em laboratório, o tratamento de frutos com $C$. laurentii foi tão ou mais eficiente na redução do diâmetro das lesões e na incidência das podridões quanto aqueles com os fungicidas thiabendazol e iprodione (Tabela 1). Nas avaliações efetuadas, constatou-se que o tratamento com a levedura reduziu significativamente a incidência (\%) e o tamanho (diâmetro em $\mathrm{mm}$ ) das lesões das podridões, quando comparado ao tratamento testemunha com os patógenos (Tabela 1).

Nos experimentos realizados em câmara fria com maçãs cv. Fuji, C. laurentii, thiabendazol, iprodione, digluconato de clorohexidina, dicloro-s-triazinatriona sódica, dicloroisocianurato de sódio e hipoclorito de sódio foram eficientes na redução do diâmetro das lesões e da incidência

TABELA 1 - Diâmetro e incidência das podridões causadas por Glomerella cingulata, Penicillium expansum e Pezicula malicorticis, em maçãs (Malus domestica) cv. Fuji

\begin{tabular}{|c|c|c|c|c|c|c|}
\hline \multirow{3}{*}{ Tratamento } & \multicolumn{6}{|c|}{ "Diâmetro da lesão (mm) } \\
\hline & \multicolumn{2}{|c|}{ G. cingulata } & \multicolumn{2}{|c|}{ P. expansum } & \multicolumn{2}{|c|}{ P. malicorticis } \\
\hline & $\mathbf{1}^{1}$ & $2^{2}$ & $\mathbf{1}^{1}$ & $2^{2}$ & $\mathbf{1}^{1}$ & $2^{2}$ \\
\hline Patógeno $\left[10^{2}\right.$ conídios $\left.\mathrm{ml}^{-1}\right](\mathrm{P})$ & $7,5 \mathrm{a}^{3}$ & $2,3 \mathrm{a}$ & $6,2 \mathrm{a}$ & $6,0 \mathrm{a}$ & $4,9 \mathrm{a}$ & $0,6 \mathrm{a}$ \\
\hline Iprodione $\left[150 \mathrm{mg} \mathrm{l}^{-1}\right]+(\mathrm{P})$ & $5,5 \mathrm{~b}$ & $1,1 \mathrm{bc}$ & $0,4 \mathrm{c}$ & $4,2 \mathrm{ab}$ & $1,7 \mathrm{~b}$ & $0,1 \mathrm{~b}$ \\
\hline Thiabendazol $\left[150 \mathrm{mg} \mathrm{l}^{-1}\right]+(\mathrm{P})$ & $5,2 \mathrm{~b}$ & $1,6 \mathrm{ab}$ & $2,6 b$ & $4,4 \mathrm{ab}$ & $1,1 \mathrm{bc}$ & $0,2 \mathrm{~b}$ \\
\hline \multirow[t]{2}{*}{ Levedura $\left[10^{7} \mathrm{ufc} \mathrm{ml}^{-1}\right]+(\mathrm{P})$} & $3,0 \mathrm{c}$ & $0,2 \mathrm{c}$ & $0,1 \mathrm{c}$ & $0,1 \mathrm{c}$ & $0,6 \mathrm{c}$ & $0,1 \mathrm{~b}$ \\
\hline & \multicolumn{6}{|c|}{ Incidência de podridão (\%) } \\
\hline Patógeno $\left[10^{2}\right.$ conídios $\left.\mathrm{ml}^{-1}\right](\mathrm{P})$ & $26,9 \mathrm{a}$ & $12,7 \mathrm{a}$ & $44,5 \mathrm{a}$ & $38,6 \mathrm{a}$ & $30,7 \mathrm{a}$ & $7,1 \mathrm{a}$ \\
\hline Iprodione $\left[150 \mathrm{mg} \mathrm{l}^{-1}\right]+(\mathrm{P})$ & $22,6 \mathrm{ab}$ & $5,8 \mathrm{bc}$ & $4,0 \mathrm{c}$ & $26,8 \mathrm{a}$ & $12,3 \mathrm{bc}$ & $0,6 \mathrm{~b}$ \\
\hline Thiabendazol $\left[150 \mathrm{mg} \mathrm{l}^{-1}\right]+(\mathrm{P})$ & $20,3 \mathrm{~b}$ & $8,1 \mathrm{ab}$ & $19,8 \mathrm{~b}$ & $28,3 \mathrm{a}$ & $7,1 \mathrm{~cd}$ & $2,1 \mathrm{~b}$ \\
\hline Levedura $\left[10^{7}\right.$ ufc ml $\left.^{-1}\right]+(\mathrm{P})$ & $14,6 \mathrm{c}$ & $1,5 \mathrm{c}$ & $0,7 \mathrm{c}$ & $0,7 \mathrm{~b}$ & $3,6 \mathrm{~d}$ & $1,5 \mathrm{~b}$ \\
\hline
\end{tabular}

${ }^{1}$ Teste $1 ;{ }^{2}$ Teste $2 ;{ }^{3}$ Os dados apresentados referem-se às avaliações efetuadas aos dez, 22 e 30 dias após a inoculação de Penicillium expasum, Glomerella cingulata e Pezicula malicorticis, respectivamente (Para maior detalhe verificar Material e Métodos); ${ }^{4}$ Médias nas colunas seguidas pela mesma letra não diferem entre si (Tukey, $5 \%$ ). No tratamento apenas com a levedura não houve desenvolvimento de lesões, portanto foi omitido da tabela por não ter sido usado na análise estatística. 
Cryptococcus laurentii aplicado em pós-colheita reduz podridões em maçãs

TABELA 2 - Diâmetro de lesões e incidência de mofo azul (Penicillium expansum), firmeza de polpa e teor de sólidos solúveis totais (SST, ${ }^{\circ}$ Brix) em maçãs (Malus domestica), cvs. Fuji e Gala, após armazenamento em câmara fria $\left(1^{\circ} \mathrm{C} / 90-95 \%\right.$ UR)

\begin{tabular}{|c|c|c|c|c|c|c|c|c|}
\hline \multirow{4}{*}{ Tratamento } & \multicolumn{6}{|c|}{ Fuji } & \multirow{2}{*}{\multicolumn{2}{|c|}{$\begin{array}{c}\text { Gala } \\
\text { Teste } 4^{4}\end{array}$}} \\
\hline & \multicolumn{2}{|c|}{ Teste $1^{1}$} & \multicolumn{2}{|c|}{ Teste $2^{2}$} & \multicolumn{2}{|c|}{ Teste $3^{3}$} & & \\
\hline & $0 \mathrm{~d}^{5}$ & $7 d^{6}$ & $\mathbf{0 ~ d}$ & $7 \mathrm{~d}$ & $\mathbf{0} \mathrm{d}$ & $7 \mathrm{~d}$ & $\mathbf{0} \mathbf{d}$ & $7 \mathrm{~d}$ \\
\hline & \multicolumn{8}{|c|}{ Diâmetro $(\mathrm{mm})$} \\
\hline Patógeno $\left[10^{2}\right.$ conídios $\left.\mathrm{ml}^{-1}\right](\mathrm{P})$ & $1,1 a^{7}$ & $6,7 \mathrm{a}$ & $0,3 \mathrm{a}$ & $4,4 \mathrm{a}$ & $6,8 \mathrm{a}$ & $16,8 \mathrm{a}$ & $0,9 \mathrm{a}$ & $4,5 \mathrm{a}$ \\
\hline Iprodione $\left[150 \mathrm{mg} \mathrm{l}^{-1}\right]+\mathrm{P}$ & $0,1 b$ & $1,3 b$ & $0,0 \mathrm{~b}$ & $2,6 b$ & $0,7 \mathrm{~b}$ & $2,7 \mathrm{~b}$ & $0,0 \mathrm{~b}$ & $0,6 \mathrm{c}$ \\
\hline Thiabendazol $\left[150 \mathrm{mg} \mathrm{l}^{-1}\right]+\mathrm{P}$ & $0,1 b$ & $0,6 \mathrm{~b}$ & $0,1 b$ & $1,1 b$ & $0,4 b$ & $1,2 \mathrm{bc}$ & $0,0 \mathrm{~b}$ & $0,0 \mathrm{c}$ \\
\hline Levedura $\left[10^{7} \mathrm{ufc} \mathrm{ml}^{-1}\right]+\mathrm{P}$ & $0,0 \mathrm{~b}$ & $0,6 b$ & $0,1 b$ & $1,4 \mathrm{~b}$ & $0,2 b$ & $0,8 \mathrm{bc}$ & $0,3 \mathrm{~b}$ & $2,9 \mathrm{ab}$ \\
\hline Dicloro-s-triazinatriona sódica $\left[150 \mathrm{mg} \mathrm{l}^{-1}\right]+\mathrm{P}$ & $*$ & * & $*$ & * & $0,9 b$ & $2,2 \mathrm{bc}$ & $0,1 b$ & $0,8 \mathrm{c}$ \\
\hline Hipoclorito de $\mathrm{Na}\left[150 \mathrm{mg} \mathrm{l}^{-1}\right]+\mathrm{P}$ & $*$ & * & $*$ & $*$ & $0,0 \mathrm{~b}$ & $1,0 \mathrm{bc}$ & $0,0 \mathrm{~b}$ & $0,8 \mathrm{c}$ \\
\hline Digluconato de clorohexidina $\left[150 \mathrm{mg}^{-1}\right]+\mathrm{P}$ & $*$ & * & * & * & $0,6 b$ & $1,2 \mathrm{bc}$ & * & $*$ \\
\hline \multirow[t]{2}{*}{ Dicloroisocianurato de $\mathrm{Na}\left[150 \mathrm{mg} \mathrm{l}^{-1}\right]+\mathrm{P}$} & $*^{8}$ & $*$ & $*$ & $*$ & * & $*$ & $0,4 \mathrm{ab}$ & $1,4 \mathrm{bc}$ \\
\hline & \multicolumn{8}{|c|}{ Incidência de podridão $(\%) 0(\%)$} \\
\hline Patógeno $\left[10^{2}\right.$ conídios $\left.\mathrm{ml}^{-1}\right](\mathrm{P})$ & $4,9 \mathrm{a}^{7}$ & $19,0 \mathrm{a}$ & $2,1 \mathrm{a}$ & $13,4 \mathrm{a}$ & $18,3 \mathrm{a}$ & $27,5 \mathrm{a}$ & $4,6 \mathrm{a}$ & $13,6 \mathrm{a}$ \\
\hline Iprodione $\left[150 \mathrm{mg} \mathrm{l}^{-1}\right]+\mathrm{P}$ & $0,7 b$ & $3,8 b$ & $0,2 b$ & $10,5 \mathrm{a}$ & $3,1 b$ & $7,8 b$ & $0,4 \mathrm{bc}$ & $3,3 b$ \\
\hline Thiabendazol $\left[150 \mathrm{mg} \mathrm{l}^{-1}\right]+\mathrm{P}$ & $0,5 b$ & $1,3 b$ & $0,3 b$ & $3,6 \mathrm{bc}$ & $1,3 \mathrm{bc}$ & $2,8 \mathrm{~cd}$ & $0,0 \mathrm{c}$ & $0,0 \mathrm{~b}$ \\
\hline Levedura $\left[10^{7} \mathrm{ufc} \mathrm{ml}^{-1}\right]+\mathrm{P}$ & $0,0 \mathrm{~b}$ & $1,8 \mathrm{~b}$ & $0,3 b$ & $5,4 \mathrm{~b}$ & $0,6 \mathrm{bc}$ & $1,7 \mathrm{~d}$ & $1,9 \mathrm{bc}$ & $11,4 \mathrm{a}$ \\
\hline Dicloro-s-triazinatriona sódica $\left[150 \mathrm{mg} \mathrm{l}^{-1}\right]+\mathrm{P}$ & $*$ & $*$ & * & * & $2,7 b c$ & $4,2 \mathrm{~cd}$ & $0,6 \mathrm{bc}$ & $3,0 \mathrm{~b}$ \\
\hline Hipoclorito de $\mathrm{Na}\left[150 \mathrm{mg} \mathrm{l}^{-1}\right]+\mathrm{P}$ & * & * & $*$ & $*$ & $0,2 \mathrm{~cd}$ & $7,2 \mathrm{bc}$ & $0,0 \mathrm{c}$ & $4,0 \mathrm{~b}$ \\
\hline Digluconato de clorohexidina $\left[150 \mathrm{mg} \mathrm{l}^{-1}\right]+\mathrm{P}$ & $*$ & * & $*$ & $*$ & $1,3 \mathrm{bc}$ & $2,2 \mathrm{~d}$ & * & $*$ \\
\hline \multirow[t]{2}{*}{ Dicloroisocianurato de $\mathrm{Na}\left[150 \mathrm{mg}^{-1}\right]+\mathrm{P}$} & $*$ & * & $*$ & * & $*$ & $*$ & $2,3 b$ & $4,4 b$ \\
\hline & \multicolumn{8}{|c|}{ Firmeza de polpa $(\mathrm{kgF})$} \\
\hline Patógeno $\left[10^{2}\right.$ conídios $\left.\mathrm{ml}^{-1}\right](\mathrm{P})$ & $6,7^{7}$ & 6,8 & 7,1 & 6,6 & 8,0 & 7,6 & 7,1 & 6,2 \\
\hline Thiabendazol $\left[150 \mathrm{mg} \mathrm{l}^{-1}\right]+\mathrm{P}$ & 6,6 & 6,9 & 6,9 & 6,8 & 7,7 & 7,3 & 7,1 & 6,3 \\
\hline Iprodione $\left[150 \mathrm{mg} \mathrm{l}^{-1}\right]+\mathrm{P}$ & 6,5 & 6,6 & 6,8 & 6,6 & 8,0 & 7,6 & 6,7 & 6,1 \\
\hline Levedura $\left[10^{7}\right.$ ufc $\left.\mathrm{ml}^{-1}\right]+\mathrm{P}$ & 6,8 & 6,5 & 6,7 & 6,5 & 7,8 & 7,4 & 7,0 & 6,3 \\
\hline Dicloro-s- triazinatriona sódica $\left[150 \mathrm{mg} \mathrm{l}^{-1}\right]+\mathrm{P}$ & $*$ & * & $*$ & * & 7,9 & 7,4 & 7,1 & 6,0 \\
\hline Hipoclorito de $\mathrm{Na}\left[150 \mathrm{mg} \mathrm{l}^{-1}\right]+\mathrm{P}$ & $*$ & * & $*$ & $*$ & 7,9 & 8,0 & 6,7 & 5,9 \\
\hline Digluconato de clorohexidina $\left[150 \mathrm{mg} \mathrm{l}^{-1}\right]+\mathrm{P}$ & $*$ & * & * & * & 7,4 & 7,3 & * & * \\
\hline \multirow[t]{2}{*}{ Dicloroisocianurato de $\mathrm{Na}\left[150 \mathrm{mg} \mathrm{l}^{-1}\right]+\mathrm{P}$} & $*$ & * & $*$ & $*$ & $*$ & $*$ & 6,7 & 6,0 \\
\hline & \multicolumn{8}{|c|}{ Teor de sólidos solúveis totais $\left({ }^{\circ}\right.$ Brix $)$} \\
\hline Patógeno $\left[10^{2}\right.$ conídios $\left.\mathrm{ml}^{-1}\right](\mathrm{P})$ & $15,6^{7}$ & 15,8 & 13,5 & 14,2 & 15,5 & 15,7 & 13,1 & 13,4 \\
\hline Thiabendazol $\left[150 \mathrm{mg} \mathrm{l}^{-1}\right]+\mathrm{P}$ & 15,4 & 15,6 & 14,1 & 14,0 & 15,4 & 15,7 & 14,2 & 14,3 \\
\hline Levedura $\left[10^{7}\right.$ ufc $\left.\mathrm{ml}^{-1}\right]+\mathrm{P}$ & 16,0 & 15,5 & 14,6 & 14,5 & 15,4 & 15,9 & 14,0 & 13,9 \\
\hline Iprodione $\left[150 \mathrm{mg} \mathrm{l}^{-1}\right]+\mathrm{P}$ & 15,4 & 15,4 & 14,0 & 14,3 & 15,5 & 15,8 & 13,9 & 14,1 \\
\hline Dicloro-s-triazinatriona sódica $\left[150 \mathrm{mg} \mathrm{l}^{-1}\right]+\mathrm{P}$ & $*$ & $*$ & $*$ & $*$ & 15,3 & 15,7 & 13,7 & 14,1 \\
\hline Hipoclorito de $\mathrm{Na}\left[150 \mathrm{mg} \mathrm{l}^{-1}\right]+\mathrm{P}$ & * & $*$ & * & $*$ & 15,3 & 15,6 & 14,0 & 14,1 \\
\hline Digluconato de clorohexidina $\left[150 \mathrm{mg} \mathrm{l}^{-1}\right]+\mathrm{P}$ & $*$ & $*$ & $*$ & $*$ & 15,2 & 15,8 & $*$ & $*$ \\
\hline Dicloroisocianurato de $\mathrm{Na}\left[150 \mathrm{mg} \mathrm{l}^{-1}\right]+\mathrm{P}$ & $*$ & $*$ & $*$ & $*$ & $*$ & $*$ & 13,5 & 13,9 \\
\hline
\end{tabular}

${ }^{1}$ Teste 1 = frutos mantidos por 60 dias em câmara fria (CF); ${ }^{2}$ Teste $2=$ frutos mantidos por 40 dias em CF; ${ }^{3}$ Teste $3=$ frutos mantidos por 90 dias em CF; ${ }^{4}$ Teste 4 = frutos mantidos por 90 dias em CF; ${ }^{5}$ Avaliação após a retirada da CF; ${ }^{6}$ Avaliação sete dias após a retirada da CF; ${ }^{7}$ Médias nas colunas seguidas pela mesma letra não diferem entre si (Tukey, $5 \%$ ); ${ }^{8 *}=$ não testado. No tratamento apenas com a levedura não houve desenvolvimento de lesões, portanto foi omitido da tabela por não ter sido usado na análise estatística.

do mofo azul ocasionado por P. expansum (Tabela 2). Nas avaliações efetuadas imediatamente e sete dias após a retirada dos frutos do armazenamento em câmara fria, a levedura reduziu a incidência e o tamanho das lesões da podridão causada por P. expansum (Tabela 2). Todavia, no experimento efetuado com a cv. Gala, a levedura reduziu significativamente os danos causados por $P$. expansum somente na avaliação efetuada imediatamente após a retirada dos frutos da câmara fria. Constatou-se também, que a aplicação de $C$. laurentii não alterou a qualidade dos frutos, característica expressa 
através das avaliações de firmeza da polpa e teor de sólidos solúveis totais ( ${ }^{\circ}$ Brix) (Tabela 2 ).

A competição por nutrientes e o hiperparasitismo podem ter sido os mecanismos de controle pois Castoria et al. (1997) relataram que $C$. laurentii agiu contra $P$. expansum e Botrytis cinerea Pers: Fr. por competição de nutrientes e por produção de altos níveis de atividade extracelular de $\beta-1,3$ glucanase na região da parede celular dos patógenos. Adicionalmente, Valdebenito-Sanhueza \& Cattanio (2001) relataram que o isolado 36 de $C$. laurentii não induziu resistência em maçã e não apresentou antibiose in vitro contra P. expansum.

São inúmeros os exemplos de antagonistas bem sucedidos em laboratório, sob condições controladas, que fracassam quando submetidos ao ambiente natural ou comercial, onde as condições de ambiente são bastante variáveis (El-Ghaouth et al., 2000; Pusey, 1999; Wilson \& Pusey, 1985). No presente estudo, o isolado 36 de $C$. laurentii mostrou-se eficiente no controle de podridão ocasionada por $P$. expansum em condições comerciais de armazenamento dos frutos, em câmara fria. Segundo Wilson \& Pusey (1985), uma dos percalços na utilização de antagonistas para o biocontrole de doenças é a dificuldade da manutenção das condições do ambiente favorável aos agentes de controle biológico. Como no presente estudo as condições de armazenamento dos frutos são constantes, os problemas apontados por Wilson \& Pusey (1985) não foram limitantes à eficiência da levedura no controle da podridão dos frutos por P.expansum.

\section{REFERÊNCIAS BIBLIOGRÁFICAS}

ABPM. Associação Brasileira dos Produtores de Maçã. http:// www.abpm.org.br/. Consulta feita em 10/9/2002.

BLUM, L.E.B., AMARANTE, C. V. T., PRADO, G., ARIOLI, C. J., GUIMARÃES, L. S. \& DEZANET, A. Cultivar, método de inoculação e concentração de inóculo afetando as podridões da maçã por Penicillium expansum e Pezicula malicorticis. Fitopatologia Brasileira 25:359-360. 2000

BONETI, J. I. S., RIBEIRO, L. G. \& KATSURAYAMA, Y. Manual de identificação de doenças e pragas da macieira. Florianópolis. EPAGRI. 1999.

CASTORIA, R., DE CURTIS, F., LIMA, G. \& DE CICCO, V. Beta1-3-glucanase activity of two saprophytic yeasts and possible mode of action as biocontrol agents against postharvest diseases. Postharvest Biology and Technology 12:293-300. 1997.

EL-GHAOUTH, A., SMILANICK, J.L. \& WILSON, C.L. Enhancement of the performance of Candida saitoana by the addition of glycolchitosan for the control of postharvest decay of apple and citrus fruit. Postharvest Biology and Technology 19:103-110. 2000.

JANISIEWICZ, W.J. \& ROITMAN, J. Biological control of Pseudomonas cepacia. Phytopathology 78:194-198. 1998.

PUSEY, P.L. Use of Bacillus subtilis and related organisms as biofungicides. Pesticide Science 27:133-140. 1989.

VALDEBENITO-SANHUEZA, R.M. \& CATTANIO, M.E. Controle biológico de Penicillium expansum em pós-colheita de maçãs 'Fuji'. Fitopatologia Brasileira 26:445. 2001. (Resumo).

WILSON, C.L. \& PUSEY, P. L. Potential for biological control of postharvest plant disease. Plant Disease 69:375-378. 1985. 